\title{
Training in Psychotherapy: A Personal View
}

\author{
Sydney Brandon, Professor of Psychiatry, University of Leicester
}

I am a strong advocate of the provision of training in psychotherapy to all psychiatrists. In my view this should comprise the enhancement of interviewing skills, development of counselling skills, understanding of and experience in the use of a variety of psychotherapeutic techniques. Among these 1 would include supportive psychotherapy, short-term psychotherapy, crisis intervention and grief counselling. The trainee should acquire some theoretical understanding of and experience in the use of behavioura methods of treatment, of social skills training and of group processes.

For many, a personal experience of close supervision in individual or group psychotherapy, or participation in an intensive residential group exercise of the ' $T$ ' group or 'TaviGroup' type, provides a valuable self-insight with some understanding of the experience of psychological stress. This could be obtained through intensive group or individual supervision of the management of two or three patients, and perhaps involvement in a two or three-week residential group experience.

Closely related to this is the need for some training in administrative psychiatry, including the use of institution or organizational structure in therapy, and the manipulation of the administrative structure of the Health Service.

I would expect many psychiatrists to be resistant to gaining experience in all of these areas in addition to all of the other training they require. Some psychiatrists would find that they had little natural skill in the use of these techniques, but most of them could be brought to an acceptable standard by further training but a few would have to be advised to recognize their deficiency when seeking a style of practice.

A few psychiatrists within the National Health Service, and as many of those who wish to embark upon private practice should have a more rigorous training in psychotherapy. For those who intend to practise within the NHS that training should be broadly based and include dynamic and behavioural psychotherapies. The more 'exclusive' psychotherapies associated with institutes or schools which espouse a single body of theory or practice and involve particularly time-consuming therapies should operate largely outside the NHS. Some broad-visioned and particularly talented 'exclusive' therapists might assume other roles within the NHS or enter into limited contractual agreements to provide teaching, supervision or direct therapy to a limited number of NHS patients with special needs.

Wherever NHS consultant psychotherapists are appointed, they should have the training of medical postgraduates as their first priority, with some involvement in institutional consultation and the support of colleagues providing general psychiatric services as second level priorities, and the education of the clergy and other non-NHS pro- fessionals as of fairly low priority. They should resist the temptation to cut themselveswoff from mainstream psychiatry and resist the spawning of more applications for more fulltime psychotherapists and senior registrars in psychotherapy at the cost of the general psychiatric service.

NHS psychotherapists do need support and encouragement from their general psychiatry colleagues, they also need a peer reference group, and this might include private practice psychotherapists and some of the multiplicity of professionals and paraprofessionals who practise various forms of psychotherapy.

The vast majority of psychiatric patients will within the foreseeable future either receive no psychotherapy or be dependent upon general psychiatrists for any psychotherapeutic help they receive. Restraints of both money and manpower make it impossible to provide 'specialist' psychotherapy for all who could be defined as in need.

We must, therefore, ensure that all psychiatrists can offer psychotherapy as part of their therapeutic armamentarium. Perhaps we need to reject or limit the practice of those trainees who are temperamentally or otherwise incapable of learning psychotherapeutic skills.

The present pressures for the registration of psychotherapists stemmed from a concern to protect the public from unscrupulous or misguided practitioners. Various meetings of interested groups have been held under august sponsorships varying from the Minister of Health to the British Association for Counselling, and the only agreement that has clearly emerged is that registration would not provide the desired protection for the public.

There are, however, very strong lobbies in favour of registration, and two of the most powerful motive forces came as quite a surprise to me. The first was for the clear identification of the profession of psychotherapy and the second for VAT exemption of the fees paid to non-medical psychotherapists. It is suggested that the first would resolve the second, but the urge towards professional independence and recognition is more powerful than a mere cash incentive.

I have no wish to impede the professional development of psychotherapists and can only admire the unity of purpose which is developing between groups as diverse as the humanistic practitioners, the transactional analysts and the Institute of Psychoanalysis. On the other hand I would strongly resist any suggestion that psychotherapy is something undertaken only by psychotherapists, or that we should have parallel (and possibly unrelated) national services for psychiatry, psychology, nursing, social work and psychotherapy.

Our first concern must be to improve the psychotherapeutic skills of psychiatrists in general as a means of providing a better service to our patients. 